\title{
Clinical profile of dyselectrolytemia in diabetic patients in ICU at admission and its correlation with outcome
}

\author{
Arun Pandey ${ }^{1, *}$, Abhinav Naithani ${ }^{2}$, Anuj Bagga ${ }^{3}$ \\ ${ }^{1}$ Senior Resident, ${ }^{2}$ Post Graduate Resident, ${ }^{3} \mathrm{PG}$ Resident, ${ }^{1}$ Doon Government Hospital and Medical College, Uttarakhand, ${ }^{2,3}$ Shri Guru \\ Ram Rai Institute of Medical \& Health Sciences, Uttarakhand, India
}

*Corresponding Author:

Email: arun_pandey09@yahoo.co.in

\begin{abstract}
Electrolyte imbalances are common problem in critically ill patients. Diabetes mellitus (DM) is one of the diseases with increased frequency of electrolyte abnormalities which can be due to various factors most commonly due to impaired renal function, acid-base disorders, malabsorption syndromes or it can be due to multidrug regimens. This study included 40 subjects admitted in the intensive care units of SGRR Institute of Medical and Health Sciences to look at the clinical profile of dyselectrolytemia in diabetic patients admitted in ICU. Hyponatremia was the most common dyselectrolytemia. Most of the patients with renal failure had hypermagnesemia and hyperkalemia. Hypertension was the most common co-morbidity.
\end{abstract}

Keywords: Diabetes mellitus, Dyselectrolytemia, Clinical profile.

\section{Introduction}

ICU is the highest mortality unit in any hospital with average mortality rate reported ranging from $8-19 \%{ }^{1}$ Electrolyte disturbances are present in around $25 \%$ of patients in ICU and contribute to overall mortality in ICU. ${ }^{2}$ Diabetes mellitus (DM) is one of the diseases with increased frequency of electrolyte abnormalities due to presence of factors like hyperglycemia, impaired renal function, malabsorption syndromes, acid-base disorders and multidrug regimens. ${ }^{3-6}$ The purpose of this study was to increase awareness of many electrolyte disturbances that can be prevented by attention to the primary illness, medications used and intravenous fluids and nutrition in ICU patients.

\section{Materials and Methods}

The study was conducted in Department of Medicine at SGRR Institute of Medical and Health Sciences, Dehradun from December 2016 to March 2017. It included 40 patients admitted in the intensive care units. The patients had type 2 diabetes mellitus presented with dyselectrolytemias, which included disturbances in serum level of sodium, potassium and magnesium, were included in the study. The patients so enrolled in the study were evaluated using a thorough clinical history in each case with special emphasis on duration of primary illness, medications used and clinical manifestations of electrolyte imbalance.

Serum electrolyte levels including $\mathrm{Na}, \mathrm{K}$ and $\mathrm{Mg}$ levels were done at the time of admission. These patients were followed for the whole duration of stay in the ICU and were observed for the serial electrolyte levels, associated comorbidities and outcome in terms of mortality. The data so obtained was analyzed by using suitable statistical methods.

\section{Results}

Out of all the patients admitted in ICU with diabetes and co-morbidities during the study period, the most common cause of admission to ICU was Acute coronary syndromes (ACS) $(42.50 \%)$ followed by Renal failure
(15\%). Sepsis (10\%), renal failure with sepsis $(7.5 \%)$ Cerebrovascular accidents CVA (7.5\%) and pneumonia (5\%) were other causes of ICU admission (Fig. 1, Table 1 to $3)$.

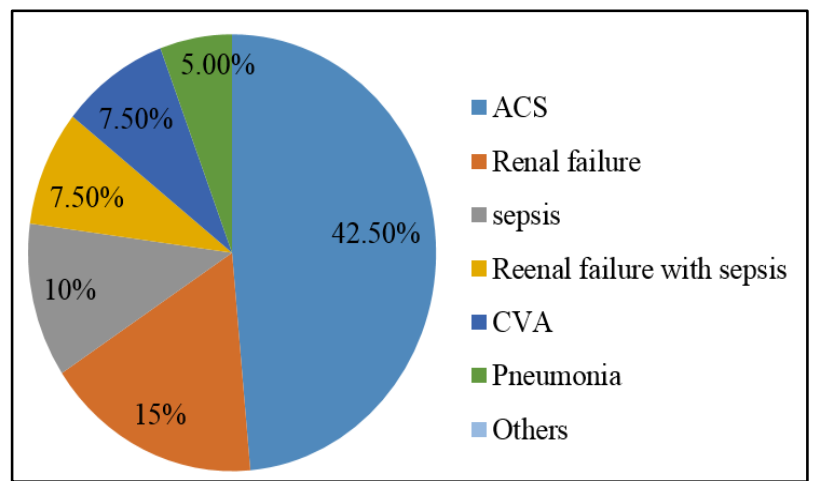

Fig. 1: Diagnosis of patients admitted in ICU

Hypertension $(57.5 \%)$ was the most common comorbidity observed in the study group. Anemia was present in $20 \%$ patients and Hypothyroidism in $12.5 \%$ patients (Fig. 2).

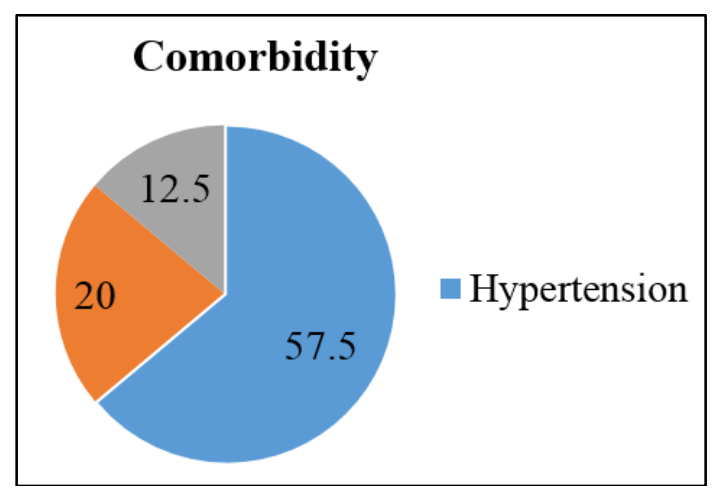

Fig. 2: Co-morbidities seen with DM 
Table 1: Pattern of dyselectrolytemia

\begin{tabular}{|c|c|c|}
\hline $\begin{array}{c}\text { Single electrolyte } \\
\text { disturbance }\end{array}$ & Two ectrolyte disturbances & All electrolytes disturbances \\
\hline 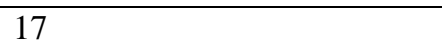 & 18 & 5 \\
\hline $\begin{array}{l}16 \text { had hyponatremia and } 1 \\
\text { had hypokalemia }\end{array}$ & $\begin{array}{l}7 \text { had hypernatremia with hypomagnesemia } \\
5 \text { had hyponatremia with hypermagnesemia } \\
2 \text { had hyponatremia with hyperkalemia } \\
2 \text { had hypernatremia with hypermagnesemia } \\
1 \text { had hypokalemia with hypermagnesemia } \\
1 \text { had hyperkalemia with hypermagnesemia }\end{array}$ & $\begin{array}{l}3 \text { had hyponatremia with } \\
\text { hypokalemia with hypomagnesemia } \\
2 \text { had hyponatremia with } \\
\text { hyperkalemia with } \\
\text { hypermagnesemia }\end{array}$ \\
\hline
\end{tabular}

Table 2: Comparison of mortality between single and multiple electrolyte disturbances

\begin{tabular}{|l|c|c|}
\hline Pattern of dyselectrolytemia & Total number & Mortality (\%) \\
\hline Total no. of dyselectrolytemia & 40 & $6(15 \%)$ \\
\hline Single electrolyte disurbance & 17 & $3(17.6 \%)$ \\
\hline Multiple electrolyte disturbance & 23 & $3(13.0 \%)$ \\
\hline
\end{tabular}

Table 3: Comparison of mortality between severity of electrolyte disturbances

\begin{tabular}{|l|c|c|}
\hline & Total number & Mortality (\%) \\
\hline Total no of dyselectrolytemia & 40 & $6(15 \%)$ \\
\hline Severe dyselectrolytemia & 3 & $1(33.3 \%)$ \\
\hline Mild to moderate dyselectrolytemia & $\begin{array}{c}2 \text { had hyponatremia }(\mathrm{Na}<120), 1 \\
\text { had hyperkalemia }(\mathrm{K}>7)\end{array}$ & $5(13.5 \%)$ \\
\hline
\end{tabular}

\section{Discussion}

Electrolyte abnormalities are common in diabetic patients and may be associated with increased morbidity and mortality. ${ }^{4}$ Factors include hyperglycemia, episodes of hypoglycemia, impaired renal function, malabsorption syndromes, acid-base disorders and multidrug regimens. ${ }^{5}$ DM is linked to both hypo- and hyper-natremia reflecting the coexistence of hyperglycemia-related mechanisms. ${ }^{6-7} \mathrm{~A}$ study by Liamis et al showed that uncontrolled DM can induce hypovolemic-hyponatremia due to osmotic dieresis. ${ }^{8}$ Beukhof CM et al proved drug-induced hyponatremia to be due to hypoglycemic agents (chlorpropamide, tolbutamide, insulin) or other medications (e.g. amitriptyline for the treatment of diabetic neuropathy). ${ }^{9,10}$ Kadowaki $\mathrm{T}$ and Moses AM et al showed that elderly patients concomitantly using diuretics have greater risk of developing hyponatremia. ${ }^{11,12}$

But in our study, out of 10 patients (all hyponatremic) taking diuretics (torsemide in 8 and furosemide in 2) only 2 patients had decrease in serial sodium concentration. Biff F. Palmer et al showed increased or normal plasma sodium concentrations in the presence of hyperglycemia as an indicator of clinically significant deficit in total body water. ${ }^{7}$ Uribarri $J$ showed in his study that incidence of hyperkalemia is higher in diabetic patients than in the general population. ${ }^{13,14}$ Chronic hyperkalemia in diabetics is most often attributable to hyporeninemic hypoaldosteronism. Many drugs that interfere with $\mathrm{K}^{+}$ excretion are associated with hyperkalemia, including angiotensin-converting enzyme inhibitors, angiotensin II receptor blockers, renin inhibitors, beta blockers and potassium-sparing diuretics. In our study 5 patients had hyperkalemia and their potassium returned to normal with drugs like Insulin and salbutamol. While 2 patients with normal potassium level at admission developed hyperkalemia without any medicine or presence of renal failure.

The causes of hypokalemia in diabetics include shift hypokalemia due to insulin administration, gastrointestinal loss of $\mathrm{K}^{+}$due to diabetic-induced motility disorders and renal loss of $\mathrm{K}^{+}$(due to osmotic diuresis and/or coexistent hypomagnesemia). The increased secretion of epinephrine due to insulin-induced hypoglycemia may also play a contributory role. ${ }^{15}$

In our study, 5 patients had hypokalemia at admission. 3 had hypomagnesemia at admission, 1 had normal and 1 had high magnesium level. All were on Insulin therapy but only 2 of them had further fall in potassium level. Hypomagnesemia is a frequent electrolyte disorder in diabetic patients. ${ }^{16}$ Recently, DM was identified as an independent risk factor for hypomagnesemia. ${ }^{2}$ Osmotic diuresis was the prominent underlying mechanism followed by diarrhea (as a result of diabetic autonomic neuropathy or metformin) and intracellular shift by insulin and epinephrine (during hypoglycemic episode). ${ }^{17-20}$ In our study 10 patients had hypomagnesemia at admission, i.e. $25 \%$, reflecting contribution of solely the disease on magnesium level. Although various studies have reported decreased levels of magnesium in type 2 diabetes patients, in our study 11 patients had hypomagnesaemia, i.e. more common than hypomagnesemia. This could be explained due to presence of renal failure in 9 patients, 1 had septic shock and other had periampullary carcinoma, both without renal failure. 


\section{Conclusion}

Mortality was $15 \%$ in our study. Hypertension was the most common co-morbidity. Hyponatremia was the most common dyselectrolytemia. Besides 2 patients, all patients with renal failure had hypomagnesaemia and 3 of 5 patients with hyperkalemia had renal failure. Severity of dyselectrolytemia seems to affect mortality. Presence of more than one electrolyte imbalance did not seem to affect mortality in this study.

\section{Conflict of Interest: None.}

\section{References}

1. Philip R. Lee. ICU Outcomes (Mortality and Length of Stay) Methods, Data Collection Tool and Data

2. Buckley MS, LeBlanc JM, Cawley MJ. Electrolyte disturbances associated with commonly prescribed medications in the intensive care unit. Crit Care Med 2010;38(Suppl.):S253-S264.

3. Elisaf MS, Tsatsoulis AA, Katopodis KP, Siamopoulos KC. Acid-base and electrolyte disturbances in patients with diabetic ketoacidosis. Diabetes Res Clin Pract 1996;34:23-27.

4. George Liamis, Evangelos Liberopoulos, Fotios Barkas, Moses Elisaf. Diabetes mellitus and electrolyte disorders. World $J$ Clin Cases 2014;2(10):488-496.

5. Elisaf MS, Tsatsoulis AA, Katopodis KP, Siamopoulos KC. Acid-base and electrolyte disturbances in patients with diabetic ketoacidosis. Diabetes Res Clin Pract 1996;34:23-27.

6. Biff F. Palmer, Deborah J. Clegg. Electrolyte and acid-base disturbances in patients with diabetes mellitus. $N$ Engl $\mathrm{J} \mathrm{Med}$ 2015;373:548-559.

7. Liamis G, Milionis HJ, Elisaf M. Hyponatremia in patients with infectious diseases. J Infect 2011;63:327-335.

8. Liamis G, Milionis H, Elisaf M. A review of drug-induced hyponatremia. Am J Kidney Dis 2008;52:144-153.
9. Beukhof CM, Hoorn EJ, Lindemans J, Zietse R. Novel risk factors for hospital-acquired hyponatraemia: a matched casecontrol study. Clin Endocrinol 2007;66:367-372.

10. Kadowaki T, Hagura R, Kajinuma H, Kuzuya N, Yoshida S. Chlorpropamide-induced hyponatremia: incidence and risk factors. Diabetes Care 1983;6:468-471.

11. Moses AM, Howanitz J, Miller M. Diuretic action of three sulfonylurea drugs. Ann Intern Med 1973;78:541-544.

12. Palmer BF. Managing hyperkalemia caused by inhibitors of the renin-angiotensin-aldosterone system. $N$ Engl J Med 2004;351:585-592.

13. Uribarri J, Oh MS, Carroll HJ. Hyperkalemia in diabetes mellitus. J Diabet Complications 1990;4:3-7.

14. Petersen KG, Schlüter KJ, Kerp L. Regulation of serum potassium during insulin-induced hypoglycemia. Diabetes 1982;31:615-617.

15. Pham PC, Pham PM, Pham SV, Miller JM, Pham PT Hypomagnesemia in patients with type 2 diabetes. Clin J Am Soc Nephrol 2007;2:366-373.

16. Liamis G, Liberopoulos E, Alexandridis G, Elisaf M. Hypomagnesemia in a department of internal medicine. Magnes Res 2012;25:149-158.

17. Svare A. A patient presenting with symptomatic hypomagnesemia caused by metformin-induced diarrhoea: a case report. Cases J 2009;2:156.

18. Paolisso G, Sgambato S, Passariello N, Giugliano D, Scheen A, D’Onofrio F, et al. Insulin induces opposite changes in plasma and erythrocyte magnesium concentrations in normal man. Diabetologia 1986;29:644-647.

19. Matsumura M, Nakashima A, Tofuku Y. Electrolyte disorders following massive insulin overdose in a patient with type 2 diabetes. Intern Med 2000;39:55-57. 